\title{
Numerical evaluation of crack stopping mechanisms in composite bonded joints due to corrugation and bolts
}

\author{
Konstantinos Tserpes \\ Laboratory of Technology $\&$ Strength of $M$ aterials, D epartment of $M$ echanical Engineering $\&$ \\ A eronautics, University of Patras, Patras, 26500, Greece
}

\begin{abstract}
In this paper, the crack stopping mechanisms in corrugated composite bonded joints and hybrid bonded/bolted joints were evaluated numerically using the cohesive zone modeling approach. For the study, the DCB (double-cantilever beam) and the CLS (crack-lap shear) specimens were modelled. The first two specimens were subjected to static loads and the latter both to static and fatigue loads. The analysis was performed using the LS-DYNA explicit FE code. Fatigue crack growth simulation was performed using an in-house developed user-defined subroutine (UMAT). The numerical results reveal a crack stopping in the corrugated $D C B$, no crack stopping in the corrugated $C L S$ and a reduction of crack growth rate in the bonded/bolted CLS for both static and fatigue loads. The methods and the findings of the present study can be used for the design of crack stopping features in adhesively bonded primary composite aircraft structures.
\end{abstract}

\section{Introduction}

While for secondary aircraft structures, adhesive bonding is a common practice both for joints and repairs, the certification rules that are applicable for primary bonded structures prevent the use of bolt-free bonded joints, as a result of earlier experiences, where the interpretation of the rules led to in-service premature failure incidents on adhesively bonded joints [1,2]. Probably the most feasible Means of Comply to achieve certification is to design Crack Stopping Features (CSFs) to limit the maximum disbond size in adhesive joints [1,2]. Recently, on 2012 a systematic research initiated on this area in the frame of the European project BOPACS (Boltless Assembling of Primary Aerospace Composite Structures) [3]. Among the CSFs examined are corrugation and bolts (chicken fasteners). Research on CSFs has been performed through tests and simulation models (virtual testing) [3-5]. In this paper, the crack stopping mechanisms in corrugated composite bonded joints and hybrid bonded/bolted joints are evaluated numerically using the cohesive zone modeling approach.

\section{Materials and specimens}


Two different specimens were modeled; a corrugated bonded double-cantilever beam (DCB) specimen and a bonded/bolted crack-lap shear specimen (CLS). The corrugated DCB was loaded by a normal quasi-static tensile load which develops a pure mode-l load at the bondline away from the corrugation while the CLS was loaded by an axial quasi-static and fatigue tensile load which develops a mixed-mode $I+1 I$ load at the entire bondline. Schematic representations and basic dimensions of the CLS specimen are shown in Fig. 1.

The adherents of the DCB specimen were made from the $R-367-2$ resin reinforced by Structil T2TE 220 fibers in $2 \times 2$ twill fabric architecture. The layup of the composite adherents is (0/90)8. The adhesive was a mixture of EA $9395(80 \%)$ and EA $9396(20 \%)$ adhesives. The adherents of the CLS specimen consists of 24 unidirectional plies of 8552/IM 7 prepreg material. The plies are stacked in the quasi-isotropic sequence of $\left[(0 / 45 / 90 /-45)_{3}\right]_{s}$. The $0.3 \mathrm{~mm}$-thick adhesive used to bond the plates is a mixture of two paste adhesive systems, namely $80 \%$ Loctite EA 9395 and 20\% Loctite EA 9396. In the bolted CLS specimen, a titanium bolt composed of a pin and a collar of diameter of $4.8 \mathrm{~mm}$ and $9.6 \mathrm{~mm}$, respectively, has been applied.

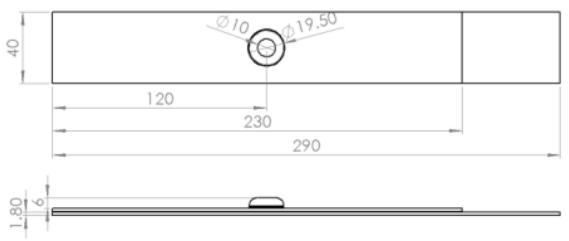

(a)

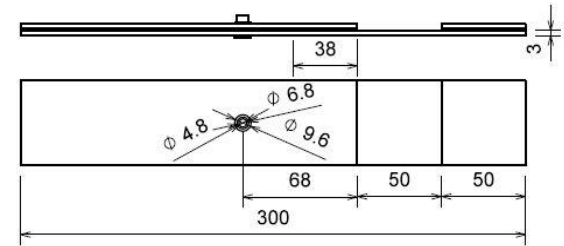

(b)

Fig. 1. Sketches and dimensions (in $\mathrm{mm}$ ) of (a) the corrugated specimen and (b) the bonded/bolted CLS specimen

\section{Simulation}

\subsection{CZM}

Quasi-static debonding initiation and growth has been simulated using the CZM method implemented in the LS-DYNA explicit FE code [6]. The CZM method is a well-established method for simulating failure of adhesive joints. For the analysis, the linear elastic/linear softening (bilinear) traction-separation law has been used. The mixed-mode behavior is described by the mixed-mode bilinear traction shown in Fig. 2(a). The ultimate displacements in the normal and tangential directions are the displacements at the time when the material has failed completely, i.e., the tractions are zero.
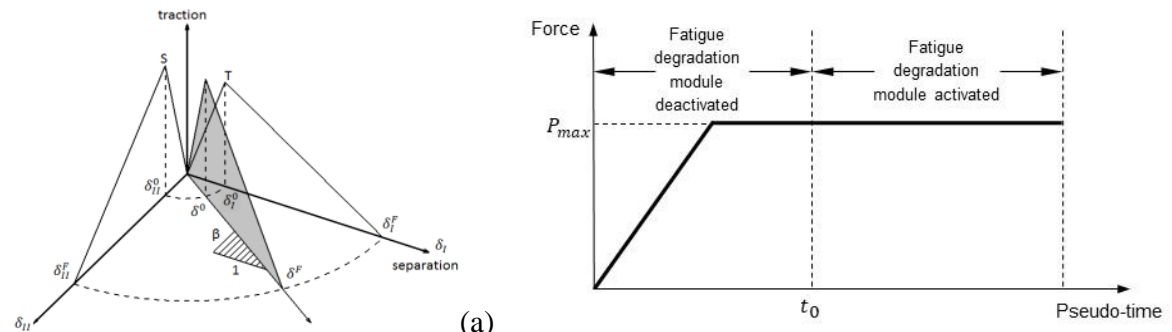

(b) 
Fig. 2. (a) The bi-linear traction separation law, (b) A pplication of load and activation of the fatigue degradation module.

Fatigue crack growth simulation in the CLS specimen has been performed through the application of constant load, as can been seen in Fig. 2(b) [7,8]. The degradation behavior of the cohesive elements has been modelled based on number of cycles using a user-defined frequency which is the number of cycles per second of the pseudo-time in LS-DYNA. The flowchart of the performed steps of the fatigue degradation module is presented in Fig. 3. The steps have been implemented through a user-defined subroutine (UMAT) developed in LS-DYNA $[7,8]$.

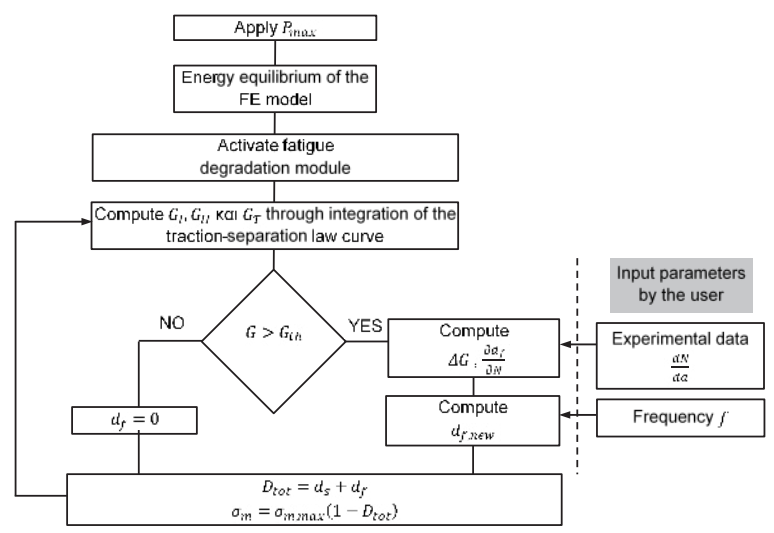

Fig. 3. Flowchart of the fatigue CZM method

\subsection{Composite failure}

For simulating damage in the composite adherents, which could be developed in the mode-II and mode-II dominated mixed-mode load-cases due to high bending loads, the progressive damage modeling method has been adopted. To this end, the material model MAT161 of the LS-DYNA has been used. The specific material model implements automatically the progressive damage modeling method by combining a set of strain-based Hashin-type failure criteria for predicting tension/shear fiber failure, compression fiber failure, perpendicular matrix failure and delamination and a damage mechanics property degradation module for simulating the damage effects.

\subsection{FE modeling}

Analyses have been performed using the LS-DYNA FE code. The FE mesh of both specimens have been created using the ANSYS Workbench platform. The composite adherents have been modeled using the Element Formulation 1 (ELFORM 1) which is an 8noded constant stress solid element with three DOFs per node. To account for damage in the composite adherents under static loading, *MAT_162_COMPOSITE_DMG of LSDYNA has been used which implements automatically the progressive damage modeling method. For the fatigue debonding growth simulation, where damage of the composite material has been not simulated, the *MAT_002_ORTHOTROPIC_ELASTIC material model has been used. Titanium bolts have been modeled using *MAT_001_ELASTIC material model. Typical FE meshes of the specimens are presented in Figs. $\overline{4}$ to $\overline{6}$. 


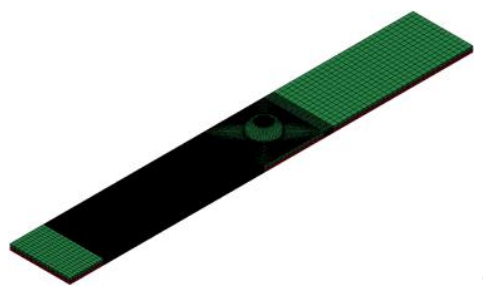

(a)

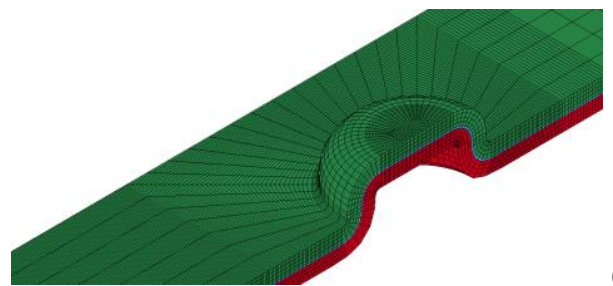

(b)

Fig. 4. (a) FE mesh of the corrugated DCB specimen, (b) FE mesh of the bolt

(a)

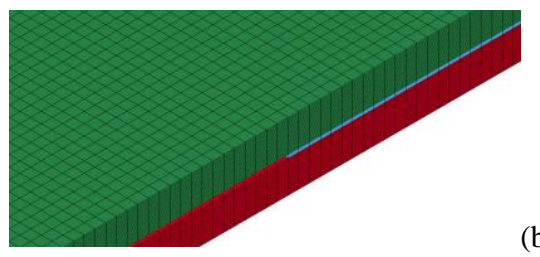

(b)

Fig. 5. (a) FE mesh of the bonded CLS specimen, (b) Close view of the FE mesh in the crack tip in the bonded CLS specimen
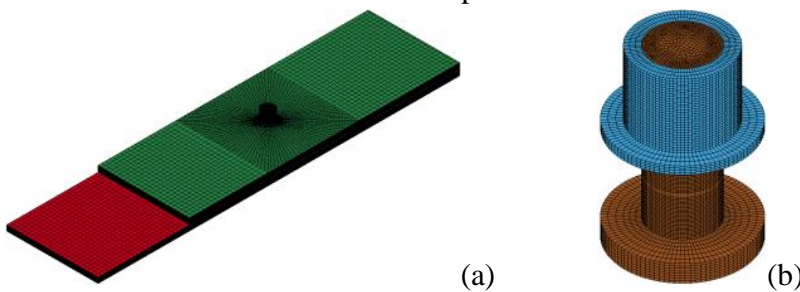

Fig. 6. (a) FE mesh of the bonded/bolted CLS specimen, (b) FE mesh of the bolt

\section{Numerical results}

\subsection{Corrugated DCB specimen}

The experimental and numerical load-displacement curves of the corrugated DCB specimen are compared in Fig. 7. The predicted debonding growth is shown in Fig. 8. In the graph, the numerical curve of the flat (no corrugation) DCB specimen has been also added. The comparison shows that the model reproduced accurately the entire experimental curve (initial stiffness, crack initiation and propagation). Debonding predicted to initiate early at the applied displacement of $0.822 \mathrm{~mm}$ at the edge of the adhesive, due to high normal stresses, and propagated towards corrugation to arrive at $21.2 \mathrm{~mm}$. At the corrugation, the debonding growth was stopped due to the shift of normal $\sigma_{z}$ stress to shear stresses. The decrease of $\sigma_{z}$ is illustrated in Fig. 9. Fig. 9(b) shows the variation of $\sigma_{z}$ at the el ement of the adhesive located at the edge of the corrugation. It is shown that when the crack reached that element (20 mm of applied displacement) $\sigma_{z}$ dropped down to -10 M Pa which led to crack stopping. The same phenomenon is also shown in Fig. 9(c) where the variation of $\sigma_{z}$ at the moving crack tip is plotted. As the crack propagated for about $120 \mathrm{~mm}$, the maximum $\sigma_{z}$ at the moving crack tip dropped from $19 \mathrm{M} \mathrm{Pa}$ ( $20 \mathrm{~mm}$ of applied displacement) down to 0.15 $\mathrm{MPa}$ (at $70 \mathrm{~mm}$ of applied displacement) which is well below the crack growth threshold. The stopping of debonding growth is reflected in the curves by the continuous load increase after $20 \mathrm{~mm}$. The numerical curve of the flat DCB specimen continues to drop after the 20 $\mathrm{mm}$ due to continuous crack growth.

Aiming to assess the effect of corrugation's geometry on crack stopping effectiveness, two additional analyses were performed: one with a corrugation of external $30 \mathrm{~mm}$ 
diameter (increased diameter) and one with a corrugation of $12 \mathrm{~mm}$ height (increased height). The predicted curves for these cases are also included in Fig. 7. In general, no major differences are observed in the predicted crack growth behavior of the two new geometries with regard to the reference corrugated geometry. There is only a higher slope in the ascending part of the curve after the $20 \mathrm{~mm}$ for both cases which is due to the higher stiffness of the corrugated area caused by the increased dimensions of corrugation.

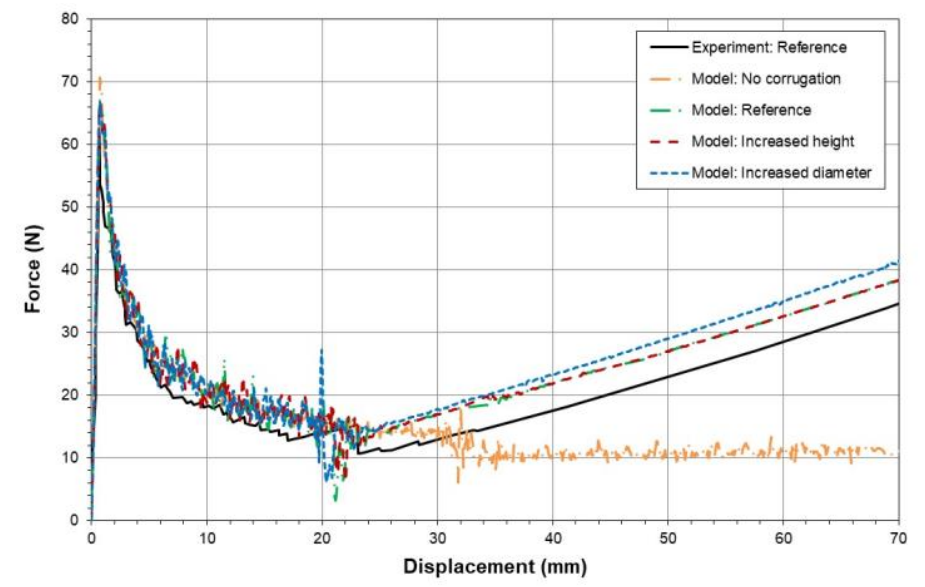

Fig. 7. L oad-displacement curves and crack propagation for the corrugated DCB specimen.

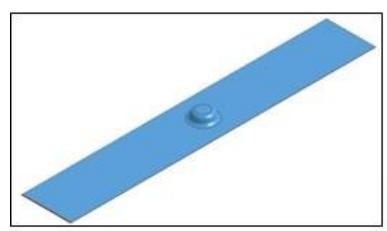

Reference: $0.58 \mathrm{~mm}$ Crack initiates at the corrugation

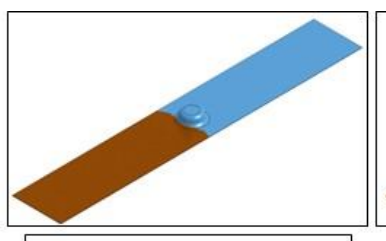

Reference: $21.2 \mathrm{~mm}$ Crack reaches the corrugation

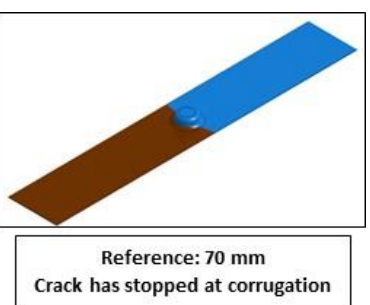

Crack has stopped at corrugation

Fig. 8. Predicted debonding growth in the corrugated specimen.
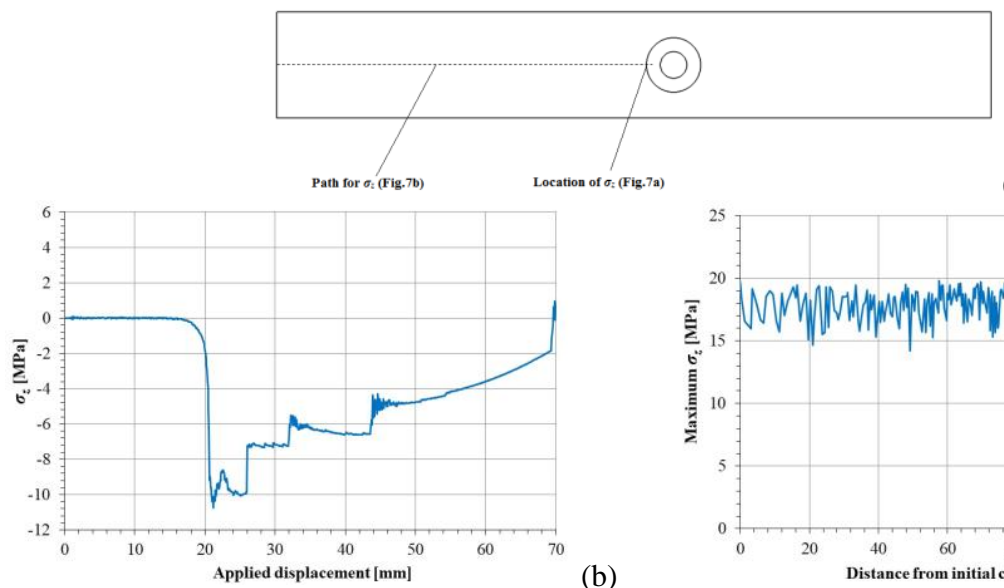

(b)

(a)

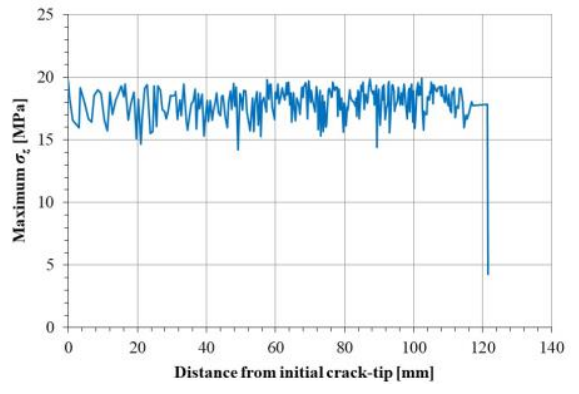

(c) 
Fig. 9. (a) L ocation of $\sigma_{z}$ for Fig. 9(b) and stress path for Fig. 9(c), (b) V ariation of normal $\sigma_{z}$ stress at the element of the adhesive located at the edge of the corrugation with regard to applied displacement,

(c) V ariation of normal $\sigma_{z}$ stress at the crack tip with regard to crack location

\subsection{Bonded/bolted CLS specimen}

Fig. 10 compares the predicted debonding growth between the bonded and bonded/bolted CLS specimens under quasi-static load. It is shown that when the crack front reaches the bolt (1.44 mm, $43 \mathrm{~mm})$, it almost stops. The sudden drop in the curve of the bonded/bolted specimen is due to failure of the composite laminate in the bolt area, which is also an important finding. The delay in debonding growth caused by the bolt is more significant and visible for fatigue loading as the fracture phenomenon is progressive. As shown in Fig. 11 , although the debonding initiates at fewer cycles in the bonded/bolted CLS specimen, when it reaches to the bolt, it slows down. The reason for the stopping of debonding growth is that the presence of bolt is causing the decrease of Iongitudinal $\sigma_{x}$ and normal $\sigma_{z}$ stresses in the adhesive which are responsible for debonding. In the contour plots of Figs. 12 and 13 , it is clearly shown that when the debonding front is reaching the bolt area, both stresses are decreasing and the debonding almost stops.

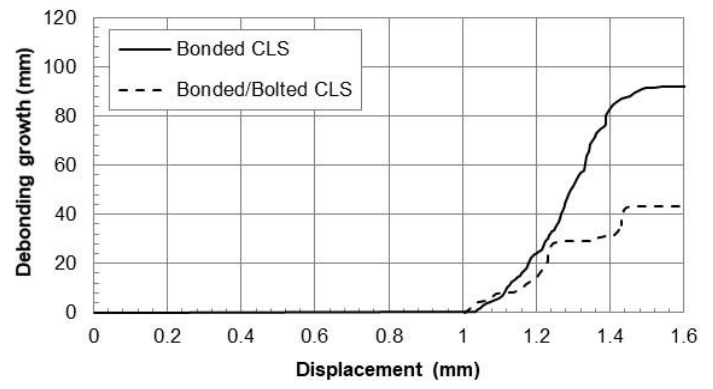

Fig. 10. Predicted debonding growth in the bonded/bolted CLS specimen.
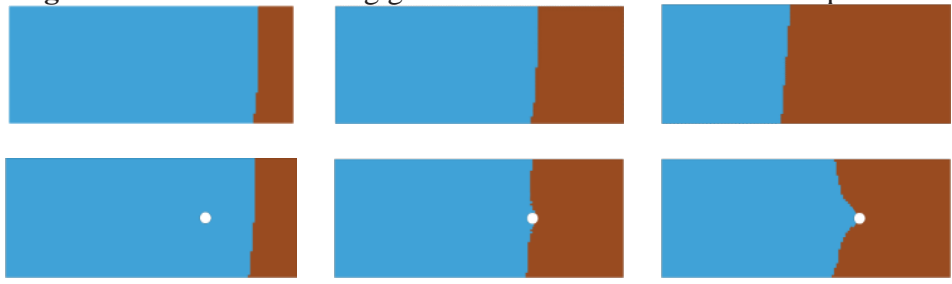

9,000 cycles

21,000 cycles

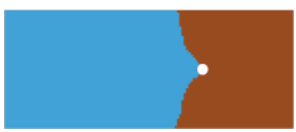

39,000 cycles

Fig. 11. Predicted fatigue debonding growth in the bonded CLS and bonded/bolted CLS specimens.
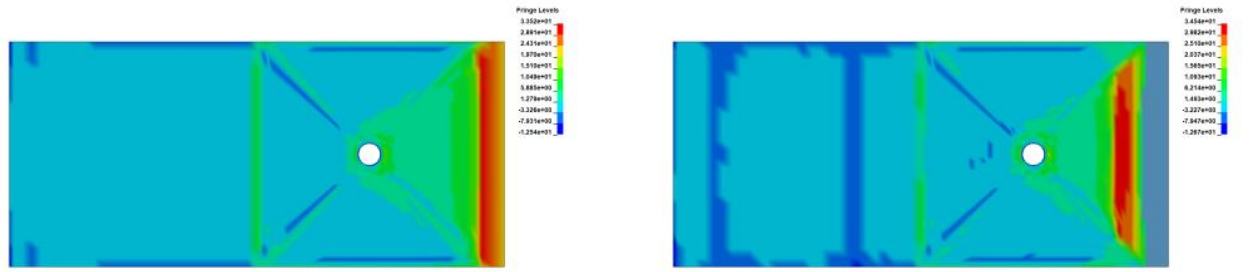

(a)

(b) 

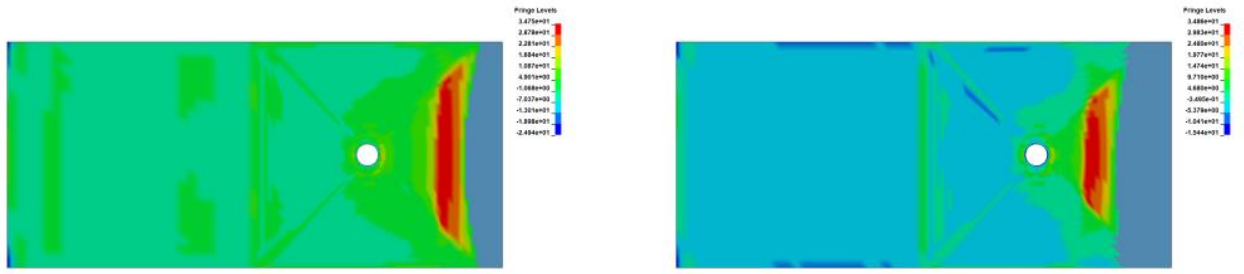

(c)
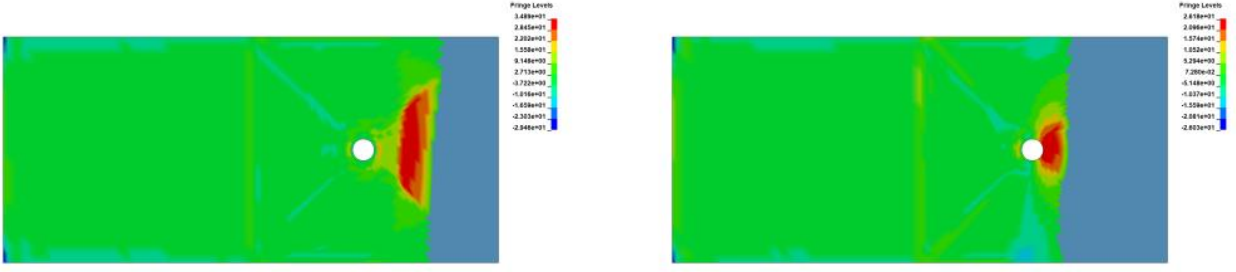

(e)

(f)

Fig. 12. Evolution of contour of longitudinal stress $\sigma_{x}$ in the adhesive of the bonded/bolted CLS specimen for different applied displacement: (a) $0.961 \mathrm{~mm}$, (b) $1.06 \mathrm{~mm}$, (c) $1.11 \mathrm{~mm}$, (d) $1.18 \mathrm{~mm}$, (e) $1.19 \mathrm{~mm}$, (d) $1.23 \mathrm{~mm}$.
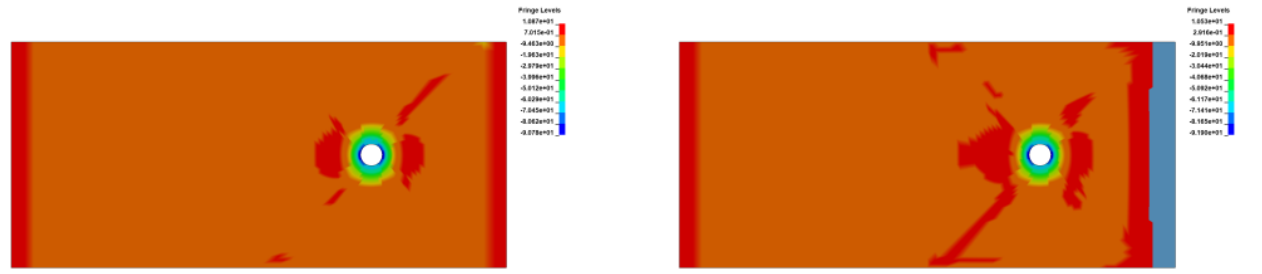

(a)

(b)
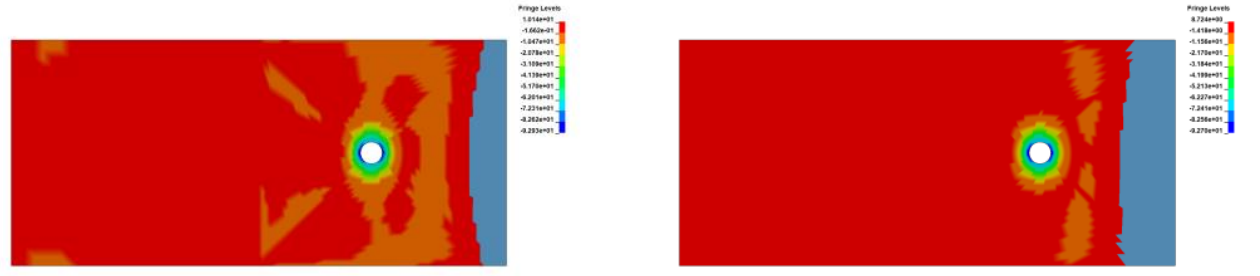

(c)

(d)
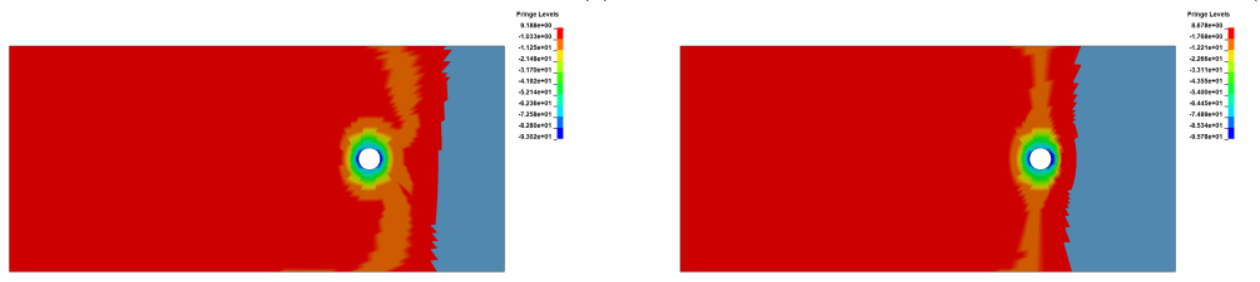

(e)

(f)

Fig. 13. Evolution of contour of normal stress $\sigma_{z}$ in the adhesive of the bonded/bolted CLS specimen for different applied displacement: (a) $0.961 \mathrm{~mm}$, (b) $1.06 \mathrm{~mm}$, (c) $1.11 \mathrm{~mm}$, (d) $1.18 \mathrm{~mm}$, (e) 1.19 $\mathrm{mm}$, (d) $1.23 \mathrm{~mm}$.

\section{Conclusions}


In this paper, the crack stopping mechanisms in corrugated DCB and hybrid bonded/bolted CLS composite joints were evaluated numerically using the CZM approach. The results verify the crack stopping capability of the corrugation and the bolt. The basic crack stopping mechanism is the reduction of normal stresses opening the crack in mode-I.

\section{References}

1. EASA. A cceptable M eans of Compliance: AM C 20-29; 2010.

2. FAA. AC 20-107B - Composite A ircraft Structure; 2010

3. Boltless joining of primary aerospace composite structures (BOPACS). A nnex-I: D escription of work. 2012.

4. K Tserpes, G Peikert, I Floros. Theoretical and A pplied Fracture M echanics, 83, 152 (2016).

5. I Floros, K Tserpes, T Löbel. Composites Part B: Engineering, 78, 459 (2015).

6. LS-DY NA theory manual. CA, USA : Livermore Software Technology Corporation; 2019.

7. K Tserpes, I Floros. Fatigue \& F racture of Engineering M aterials \& Structures, 42, 1430 (2019).

8. I Floros, K Tserpes. Composite Structures, 207, 531 (2019). 